EPJ Web of Conferences 116, 01005 (2016)

DOI: $10.1051 /$ epjconf/201611601005

(C) Owned by the authors, published by EDP Sciences, 2016

\title{
The effects of Earth's magnetic field on 3-inch diameter photomultipliers used in KM3NeT neutrino telescope
}

\author{
V. Giordano ${ }^{\text {a }}$, S. Aiello, E. Leonora, and N. Randazzo on behalf of the KM3NeT Collaboration \\ INFN sezione di Catania, via Santa Sofia 64, 95125 Catania, Italy
}

\begin{abstract}
The KM3NeT neutrino telescope will be the largest underwater neutrino telescope and will be located in the abyss of the Mediterranean Sea. In neutrino telescopes the key element of the detector is the optical module and for KM3NeT it consists of 31 PMTs stored inside a transparent pressure-resistant glass sphere of 17-inch that serves as mechanical protection while ensuring good light transmission. Since the PMTs installed into an underwater neutrino telescope can change their orientation because of movements of the detector structure due to sea currents, the influence of Earth's magnetic field has been investigated. Magnetic shielding by means of a mu-metal cage is used to reduce magnetic effects and to make the response of the PMT sufficiently orientation independent. In order to quantify the effect on magnetic field, we compared measurements on variation of gain, transit time spread and detection efficiency for a 3-inch PMT in shielded and unshielded condition at 3 PMT inclinations. Data shows that variations are sufficiently low especially for timing properties.
\end{abstract}

\section{Introduction}

The KM3NeT Observatory [1] is a large scale neutrino telescope to be built in the deep waters of the Mediterranean Sea. In neutrino telescope the key element of detection is the optical module that consists of one or more photomultipliers (PMTs) stored inside a transparent pressure-resistant glass sphere that serves as mechanical protection while ensuring good light transmission. The KM3NeT collaboration has developed an innovative design of an optical module composed by 31 PMTs of 3-inch diameter and the readout electronics housed together in a 17-inch glass sphere (Digital Optical Module-DOM) [2]. Since PMTs are installed into an underwater neutrino telescope, they can change their orientation because of the movements of the detector structure due to sea currents so the influence of Earth's magnetic field should be investigated. Indeed the performance of a PMT could be subject to significant variation due to magnetic fields. Detection efficiency, transit time spread (TTS) and gain were measured simultaneously while varying the PMT's orientations with respect to the Earth's magnetic field, both with and without a mu-metal cage as magnetic shielding.

\footnotetext{
a e-mail: valentina.giordano@ct.infn.it
}

This is an Open Access article distributed under the terms of the Creative Commons Attribution License 4.0, which permits unrestricted use, distribution, and reproduction in any medium, provided the original work is properly cited. 


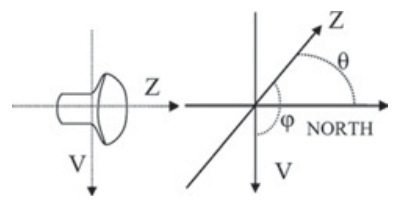

Figure 1. On the left: PMT axis (Z) and vertical axis (V). On the right: angle of inclination $\phi$ (zenith angle) and angle of rotation $\theta$ (azimuth angle).

\section{Experimental setup}

We tested one PMT with a 3-inch photocathode diameter model R12199-02 produced by Hamamatsu [3]. In the laboratory used for these measurements, the value of the magnetic field was locally measured and its value was about $40 \mu$ Tesla with good uniformity over $1 \mathrm{~m}$ of area. In order to characterize the PMT response to an injected light source while varying its orientation relative to the Earth's magnetic field, a light-tight dark box that can rotate with respect to vertical axes (step of $1^{\circ}$ ) and to change its inclination (step of $10^{\circ}$ ) was constructed. Orientation of the PMT is shown in Fig. 1. It is possible to shield a 3-inch PMT against the magnetic field using a passive magnetic shield. The shield used in this work was a wire cage, made of $1 \mathrm{~mm}$ diameter wire of mu-metal, a nickel-iron alloy with very high relative magnetic permeability of $10^{5}$. The cage was composed of two parts: an hemispherical part (which surrounds the entire PMT) and a flat part. The pitch of the grid was $(68 \cdot 68) \mathrm{mm}^{2}$, giving a shadow effect on the photocathode of less than $1 \%$ of the cathode area. The reduction factor of the magnetic field inside the cage was about 4. A laser source of $410 \mathrm{~nm}$ wave length and 60 ps pulse width was used to illuminate the photocathode of the PMT in the dark box. The variables measured changing the orientation $(\theta, \Phi)$ were: transit time (TT), transit time spread (TTS), gain and detection efficiency, defined as the ratio between the number of detected pulses and those emitted by the laser. For the detection efficiency a second PMT was used to count photons of the laser source. In order to compare results obtained with the different photomultipliers, the PMT started its rotation from the same position with respect to the box and to the Earth's magnetic field. The PMT was measured in three inclinations of the box: horizontally oriented $\left(\Phi=90^{\circ}\right), 50^{\circ}$ downwards and $50^{\circ}$ upwards. For each inclination, the PMT under test was rotated over $360^{\circ}$ in steps of $45^{\circ}$. All measurements were made first with the PMT unshielded and then repeated with the mu-metal magnetic shield. The PMT was powered by a passive base.

\section{Results}

For all sets of measured parameters the minimum, maximum, average values and maximum variation $(($ max.value - min.value $) /$ min.value $)$ are calculated for the unshielded and shielded condition of the PMT. Two examples of the behaviour of the detection efficiency and gain variations with $\theta$ at constant $\Phi=-50^{\circ}$ are shown in Fig. 2 where the blue lines represent the unshielded condition and the red lines the shielded ones. As shown in Fig. 2, higher fluctuations in gain and detection efficiency are found with rotations in the unshielded (naked) condition, while in the shielded condition the variation with $\theta$ is smaller. In Tables 1, 2 and 3 these values are reported for each inclination $\Phi$ of dark box and for the two conditions of PMT, unshielded and shielded. The measured values for the relative transit time are not reported in tables since the maximum variation found for PMT was less than $1 \%$ in unshielded condition, i.e the relative TT is only weakly dependent on the magnetic field. Values of the TTS in full width half maximum (FWHM) are shown on Table 1. We find that also TTS seems to be weakly affected by the magnetic field since generally its maximum variation on unshielded condition has a maximum of $8.10 \%$ reaching $5.01 \%$ with the mu-metal cage. Time properties can be affected by the geometry of the 


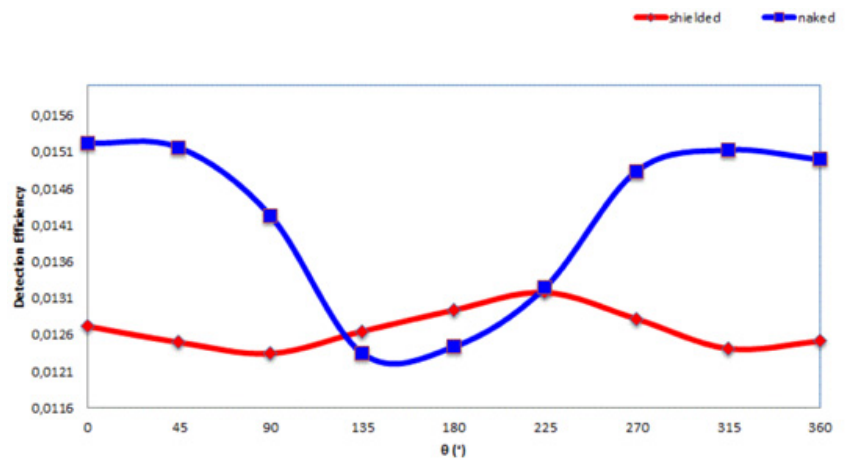

a)

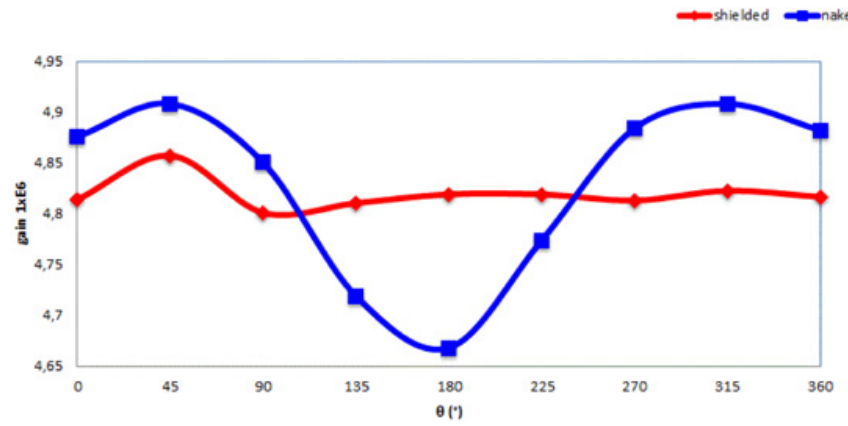

b)

Figure 2. Detection Efficiency (a) and Gain (b) for a 3-inch PMT, $360^{\circ}$ rotated, with an inclination $\Phi=-50^{\circ}$ in shielded (red line) and unshielded condition (blue line).

Table 1. Transit Time Spread Measurements.

\begin{tabular}{l|ll|ll|ll}
\hline TTS FWHM (ns) & \multicolumn{2}{|l|}{$\Phi=90^{\circ}$} & \multicolumn{3}{|l|}{$\Phi=-50^{\circ}$} & \multicolumn{2}{l}{ } & \multicolumn{2}{l}{} & \\
\hline \hline & unshielded & shielded & unshielded & shielded & unshielded & shielded \\
\hline Minimum Value (ns) & 3.64 & 3.55 & 3.63 & 3.52 & 3.58 & 3.49 \\
\hline Maximum Value (ns) & 3.79 & 3.61 & 3.75 & 3.58 & 3.87 & 3.67 \\
\hline Average Value (ns) & 3.72 & 3.58 & 3.71 & 3.55 & 3.71 & 3.59 \\
\hline Maximum Variation [\%] & 4.04 & 1.69 & 3.30 & 1.49 & 8.10 & 5.01 \\
\hline
\end{tabular}

PMT, as has been reported for 10-inch PMTs [4]. We can say that in a 3-inch PMT, time properties are not highly influenced by the effect of the natural magnetic field. Concerning the gain of the PMT, Table 2 shows that the maximum variation was not higher than 5.1\%. It should be noticed that the base voltage partition of PMTs will change in the final DOM configuration, so the voltage between the photocathode and the first dynode will change value. Moreover the final nominal gain will be of $3 \cdot 10^{6}$ instead than $5 \cdot 10^{6}$ but for these sets of measurements of gain we can say that the effects of the magnetic field are low and not as high as for the large area PMTs [4]. Concerning the measurements of the detection efficiency in Table 3, it seems to be the parameter with higher variation between shielded and unshielded condition. The largest deviation is measured for $\Phi=-50^{\circ}$ in which unshielded variations are at a maximum of $23.30 \%$. However also in this case the mu-metal cage turns out to reduce variations reducing maximum variation to the value of $6.75 \%$. 
Table 2. Gain Measurements.

\begin{tabular}{l|ll|ll|ll}
\hline Gain & \multicolumn{2}{|l|}{$\Phi=90^{\circ}$} & \multicolumn{2}{|l|}{$\Phi=-50^{\circ}$} & \multicolumn{2}{l}{$\Phi=50^{\circ}$} \\
\hline \hline & unshielded & shielded & unshielded & shielded & unshielded & shielded \\
\hline Minimum Value $\left[10^{6}\right]$ & 4.59 & 4.75 & 4.67 & 4.77 & 4.63 & 4.77 \\
\hline Maximum Value $\left[10^{6}\right]$ & 4.72 & 4.83 & 4.91 & 4.86 & 4.80 & 4.90 \\
\hline Average Value $\left[10^{6}\right]$ & 4.67 & 4.78 & 4.83 & 4.81 & 4.72 & 4.82 \\
\hline Maximum Variation $[\%]$ & 2.98 & 1.62 & 5.10 & 1.79 & 4.10 & 2.83 \\
\hline
\end{tabular}

Table 3. Relative Detection Efficiency Measurements.

\begin{tabular}{|c|c|c|c|c|c|c|}
\hline Rel. Detection Eff. & \multicolumn{2}{|l|}{$\Phi=90^{\circ}$} & \multicolumn{2}{|l|}{$\Phi=-50^{\circ}$} & \multicolumn{2}{|l|}{$\Phi=50^{\circ}$} \\
\hline & $\overline{\text { unshielded }}$ & shielded & $\overline{\text { unshielded }}$ & shielded & unshielded & shielded \\
\hline Minimum Value $\left[10^{-3}\right]$ & 11.86 & 12.34 & 12.34 & 12.34 & 12.34 & 11.90 \\
\hline Maximum Value $\left[10^{-3}\right]$ & 13.28 & 13.17 & 15.22 & 13.17 & 13.11 & 13.20 \\
\hline Average Value $\left[10^{-3}\right]$ & 12.58 & 12.36 & 14.17 & 12.67 & 12.35 & 12.48 \\
\hline Maximum Variation [\%] & 11.93 & 6.76 & 23.30 & 6.75 & 11.90 & 11.10 \\
\hline
\end{tabular}

\section{Conclusions}

The KM3NeT collaboration aims at constructing a cubed kilometer sized telescope in the abyss of the Mediterranean Sea using optical modules comprising: 31 PMTs of 3-inch stored in a glass sphere of 17-inch. Since the orientation of photomultipliers is crucial, we have investigated the possible effects of the Earth's magnetic field on the performance of the PMT. We choose as dark box for testing the 3 -inch PMT, a dark box which can rotate in $\theta$ and $\Phi$. We chose 3 inclinations of $\Phi$ and we variate $\theta$ at steps of $45^{\circ}$. We shield PMT from magnetic field using a mu-metal cage and we measure several PMT's quantities at several angles determining their variations. We investigated variations on time properties of the PMT (TT and TTS) and found no significant variation. The maximum variation on the gain was also very low for the 3-inch PMT, while higher variations were found for the detection efficiency. Using a mu-metal shielding, the variations were drastically reduced for all the quantities measured. For the 3inch PMTs the impact of the magnetic field was found to be smaller than those of large area PMTs. The larger effect was found on detection efficiency but not so high to justify the use of a magnetic shielding in the DOM made with 3-inch PMTs.

\section{References}

[1] KM3NeT (2010), ISBN 978-90-6488-033-9, www . km3net . org/TDR/TDRKM3NeT . pdf

[2] S.A. Martinez et al., The European Physical Journal C 74 (2014)

[3] Hamamatsu photonics web site (2015), https : //www . hamamat su.com

[4] S. Aiello et al., IEEE Transaction on Nuclear Science 59, issue 4 (2012) 